\title{
A Medical Assistant-Based Program to Pro- mote Healthy Behaviors in Primary Care
}

\author{
Robert L. Ferrer, MD, MPH \\ Priti Mody-Bailey, MD, $M A^{1,2}$ \\ Carlos Roberto Jaén, MD, $P b D^{1}$ \\ Sherrie Gott, $P b D^{1}$ \\ Sara Araujo, $B A^{3}$ \\ 'Department of Family and Community \\ Medicine, University of Texas Health Sci- \\ ence Center at San Antonio, San Antonio, \\ Texas \\ ${ }^{2}$ Community Medicine Associates, San \\ Antonio, Texas
}

${ }^{3}$ University of New Mexico School of Public Health, Albuquerque, New Mexico

ATC

Annals Journal Club selection, see inside back cover or http://www. annfammed.org/AJC/.

Conflicts of interest: none reported

\section{CORRESPONDING AUTHOR}

Robert L. Ferrer, MD, MPH Department of Family and Community Medicine

University of Texas Health Science Center at San Antonio

7703 Floyd Curl Dr

San Antonio, TX 78229-3900

FerrerR@uthscsa.edu

\begin{abstract}
PURPOSE Most primary care patients have at least 1 major behavioral risk: smoking, risky drinking, low physical activity, or unhealthy diet. We studied the effectiveness of a medical assistant-based program to identify and refer patients with risk behaviors to appropriate interventions.
\end{abstract}

METHODS We undertook a randomized control trial in a practice-based research network. The trial included 864 adult patients from 6 primary care practices. Medical assistants screened patients for 4 risk behaviors and applied behaviorspecific algorithms to link patients with interventions. Primary outcomes were improved risk behaviors on standardized assessments. Secondary outcomes included participation in a behavioral intervention and the program's effect on the medical assistants' workflow and job satisfaction.

RESULTS Follow-up data were available for $55 \%$ of participants at a mean of 12 months. The medical assistant referral arm referred a greater proportion of patients than did usual care (67.4 vs $21.8 \% ; P<.001)$ but did not achieve a higher success rate for improved behavioral outcomes ( 21.7 vs $16.9 \%$; $P=0.19$ ). Qualitative interviews found both individual medical assistant and organizational effects on program adoption.

CONCLUSION Engaging more primary care team members to address risk behaviors improved referral rates. More extensive medical assistant training, changes in practice culture, and sustained behavioral interventions will be necessary to improve risk behavior outcomes.

Ann Fam Med 2009;7:504-512. doi:10.1370/afm.1059.

\section{INTRODUCTION}

S moking, risky drinking, physical inactivity, and unhealthy diet are pervasive challenges to health across the world. ${ }^{1,2}$ With its high population contact rate, ${ }^{3,4}$ primary care is well-positioned to address unhealthy behaviors, but its historical development as a physician-centered acute care delivery system has hampered its ability to manage the large burden of unhealthy behaviors and the chronic diseases that follow. ${ }^{5}$ Most practices confront behavioral risks with a very limited response set-physician assessment and management—creating a bottleneck limited by the flow of physician workload. ${ }^{6,7}$ Better outcomes call for a model of primary care that manages population risk at the necessary scale. ${ }^{8}$ Such management requires engaging other members of the primary care team and forging linkages with community resources that can amplify the practice's impact. ${ }^{9}$

A framework for managing behavioral risks in primary care emerges from the concept of multiscale complexity. ${ }^{10}$ Organizations that address complicated problems must perform an array of tasks that vary in complexity and scale. Complexity measures the degree to which tasks require independent judgment and customization to produce a good outcome. Scale quantifies the number of tasks to be performed. An important principle in optimizing efficiency, therefore, is to correctly design a system that can respond to its environment with both low-variety and low-complexity tasks delivered on a 
large scale, and high-variety and high-complexity tasks delivered on a small scale. ${ }^{10,11}$ In managing behavioral risks, this framework suggests an approach to the 5 As (assess, advise, agree, assist, arrange ${ }^{12}$ ) that combines universal screening of the practice population for risk behaviors with the capacity for a high volume of lowintensity advising, as well as resources for more intensive counseling for patients who require it.

In this study, we tested a portion of this model, combining universal practice-based screening for health behaviors with low-intensity management by medical assistants (MAs), reserving certain higher complexity elements for clinician management. Organizing our program around MAs leverages an important human resource. MAs spend considerable time with patients, averaging about one-half as much time with them as physicians do. ${ }^{13,14}$ And MAs have successfully participated in other prevention programs, including mammography referral, ${ }^{15}$ tobacco cessation, ${ }^{16,17}$ and screening for domestic violence. ${ }^{18}$ Recent proposals to transform primary care highlight the crucial importance of redefining practice roles to optimize teamwork and appropriate application of skills..$^{19,20}$

\section{METHODS}

\section{Overview}

We compared a medical assistant-driven program of screening and referral for 4 health behaviors with usual care. This randomized trial was funded in round 2 in the Prescription for Health Program of the Robert Wood Johnson Foundation and Agency for Healthcare Quality and Research. ${ }^{21}$

\section{Setting and Population}

We recruited 864 patients from 6 primary care practices in the practice-based research network of San Antonio (PRENSA). These urban safety-net clinics serve a large, uninsured population that receives care through a county assistance program (CareLink), which provides comprehensive medical care at discounted rates on a sliding repayment scale. Patients were eligible if they were aged 18 years or older, covered by CareLink, and visiting for chronic disease care or health maintenance. Patients were recruited by the MAs at their discretion so as not to disrupt patient flow during peak patient care periods. Tracking patient refusals to participate did not prove to be feasible, so we assessed the representativeness of participating patients by comparing participants' demographics with those of the target clinical population.

\section{Procedure}

Behavioral risk factors were assessed through a 2step mechanism. While the MAs were measuring the patients' blood pressure and temperature, they asked all patients 2 questions to screen for smoking (Do you currently smoke? How many cigarettes or packs per day?) and 1 to screen for risky drinking (How much alcohol do you drink?), and they measured the patients' height and weight. If patients answered affirmatively to smoking, consumed alcohol above threshold levels ( 2 drinks a day for men or 1 a day for women) or had a body mass index of $25 \mathrm{~kg} / \mathrm{m}^{2}$ or greater (to screen for poor diet or physical inactivity), MAs administered a standardized behavioral risk assessment, available in either English or Spanish. The assessment included tobacco use items from the Society for Research on Nicotine and Tobacco, ${ }^{22}$ alcohol use items from the Behavioral Risk Factor Surveillance System questionnaire, ${ }^{23}$ the International Physical Activity Questionnaire (IPAQ), ${ }^{24}$ and the Starting the Conversation: Diet (STC-Diet) instrument. ${ }^{25}$

Baseline questionnaires also included demographics and the Center for Disease Control and Prevention (CDC) Healthy Days measure of physical and emotional health in the past 30 days. ${ }^{26}$

After completing their assessment, MAs assigned patients to either the MA assessment and referral or control arms according to whether the last digit of the medical record number was odd or even. MAs then informed patients in the treatment arm about their risk behaviors and asked whether they were interested in addressing a behavior; if so, patients with more than 1 risky behavior were asked to choose which to address first. Patients selected a risk behavior before being told about specific treatment options. The rationale for having patients choose their behavior was rooted in behavior change theory ${ }^{27}$ and empirical evidence that patients may be overwhelmed by multiple messages. ${ }^{28}$

The algorithm MAs used to assess behaviors and suggest referrals is shown in Figure 1. MAs were not expected to counsel for behavior change beyond encouraging patients to participate in an intervention. Interventions were available within the practice, health system, and community. Smokers could choose either a tobacco quit line ${ }^{29}$ or an 8 -week smoking cessation class offered by the county health system. Physically inactive patients could choose a community-based walking program $^{30}$ or a supervised 8 -week low-impact aerobics program in the health system (the patient's clinician had to approve the appropriateness of an exercise referral). Those with unhealthy diet could attend a 6-session healthy cooking class. ${ }^{31}$ Because of concerns that MAs could not sensitively address risky drinking during their brief time with patients, MAs did not make alcohol treatment referrals; instead, they informed clinicians about patients' drinking status. Clinicians could then, based on their own clinical assessment, choose to pro- 
vide brief office counseling, ${ }^{32}$ refer to Alcoholics Anonymous or a substance abuse clinic, or not intervene.

The control group received usual care for their risk behaviors, meaning that after the MA assessment, they visited their clinician without MA referral. Clinicians did not receive the results of the risk behavior screening. Four practices had preexisting programs to address smoking: MAs recorded smoking status as a vital sign (though they did not recommend referrals), and clinical teams' counseling for tobacco cessation was tracked and rewarded by a pay-for-performance program.

\section{Staff Training}

The assessments and referrals were performed by certified MAs, none of whom held nursing or other advanced certifications. MAs attended three 1-hour training sessions at which research staff described the project goals and details of the referral algorithm. MAs also practiced scripts for informing patients about their behavioral risks and encouraging them to choose a health risk behavior for attention. Training emphasized the need to avoid bias in selecting patients, not sway a patient's choice of health risk behavior, and not intervene in the control group.

\section{Outcomes}

The primary outcome was change in risk behaviors. For analytical purposes, we set threshold levels for behaviors at the following levels. For smoking, the patient had smoked at least 100 cigarettes in lifetime and smoked in past 7 days. For risky drinking, the patient was drinking more than 14 drinks per week for men and 7 drinks per week for women, or the patient reported any binge drinking in past month (5 or more drinks on 1 occasion). For low physical activity, the patient was categorized as "inactive" by IPAQ algorithm. For unhealthy diet, the patient consumed fewer than 5 servings of fruits and vegetables per day on the corresponding item from the STC-Diet instrument. A favorable outcome was defined as moving from above to below the thresholds.

Prespecified secondary outcomes included the proportion of completed referrals (assessed by patient report), a time-motion assessment of the MA workload required to assess and refer patients, and a qualitative assessment of the program's effects on MA job satisfaction.

\section{Follow-up Assessments}

We assessed outcomes by readministering the standardized risk assessment during a follow-up clinic visit or by telephone, $88 \%$ were completed by telephone by trained research associates. Ten attempts were made to contact participants before they were considered lost to follow-up. Although we planned to survey participants again 6 to 9 months after enrollment, median time to follow-up was 360 days, with a range of 159 to 565 days; the 10th and 90th percentiles were 215 and 441 days. Because of this wide range, we explored whether the behavioral outcomes differed by follow-up interval, but observed no statistically significant differences.

\section{Figure 1. Algorithm for medical assistants' risk behavior assessment and management.}

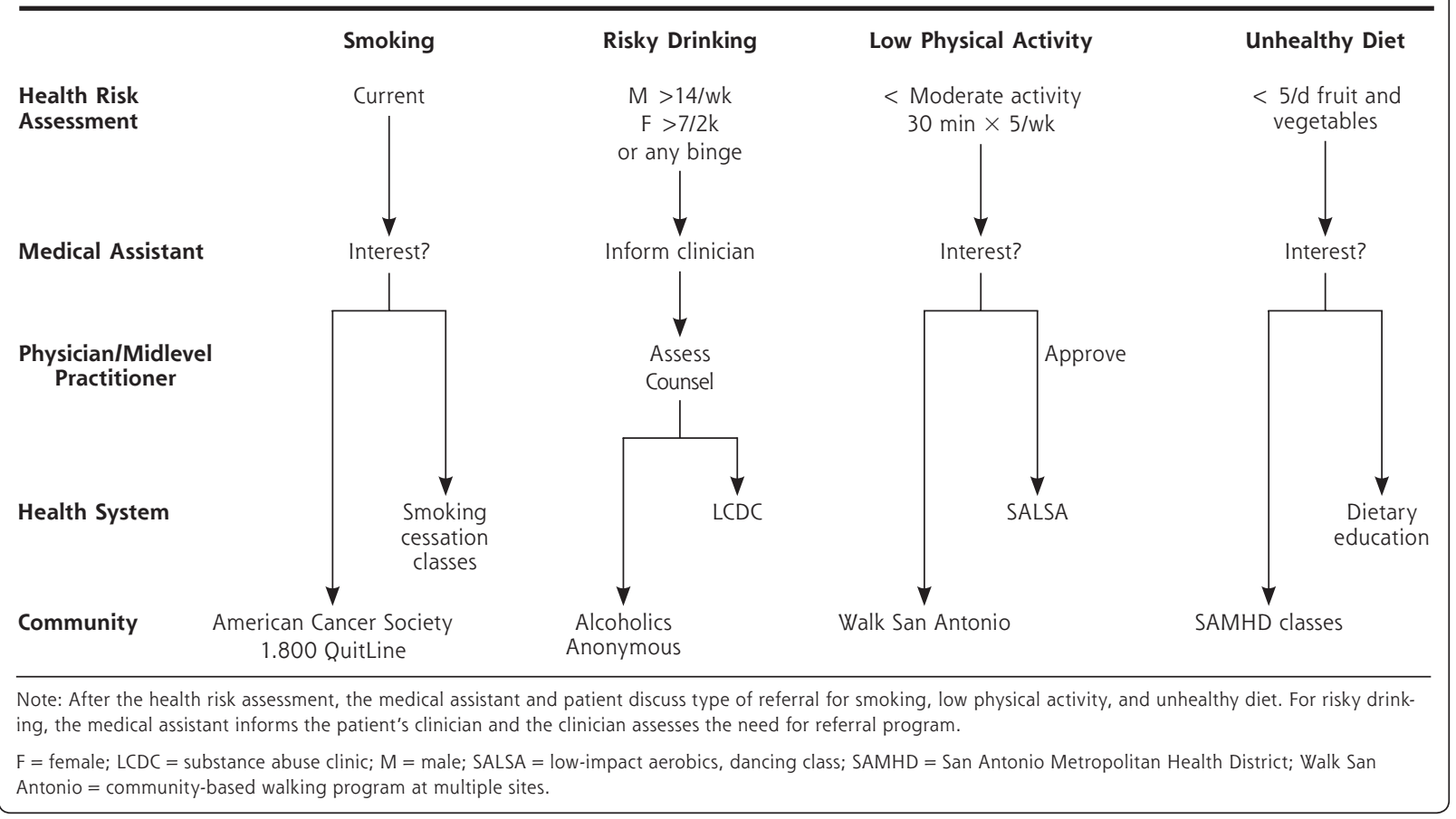




\section{Analysis}

Because control group patients did not select a risk behavior for intervention, a unique control group for each risk behavior did not exist; therefore, we applied the following analytical strategy. Referral group participants were analyzed by the risk behavior they selected for intervention. In contrast, control group participants were included in the analysis for each behavior they engaged in. For example, a control group smoker who was physically inactive would be included in the control group for both behaviors. Prestudy power calculations indicated that approximately 1,450 patients were needed per subset to detect a $3 \%$ to $5 \%$ absolute difference between groups in the proportion of patients with a specific behavioral risk.

Proportions of referral and control patients who improved a behavior or attended an intervention were compared with a 2 -sample test of proportions. Because the groups differed slightly in health status, and better educated patients who were not of a minority ethnicity were more likely to complete follow-up, we used multivariate logistic regression to adjust outcomes for demographic covariates, health status, and treatment allocation. As behavior change was a common outcome, we computed risk ratios $^{33}$ rather than odds ratios.

Risk behavior summary variables were missing values for $6.7 \%$ of the smoking sample, $22.2 \%$ of the risky drinking sample, $12.1 \%$ of the unhealthy diet sample, and $42.4 \%$ of the low physical activity sample. Women and those with less education were more likely to have missing values. In particular, respondents with fewer years of education had a high nonresponse rate on the IPAQ items asking the number of minutes per day spent performing a specified category of physical activity. Given the random pattern of missing values, we imputed them using multiple imputation Monte Carlo methods. ${ }^{34} \mathrm{We}$ used the data set with imputations to calculate the prevalence of risk behavior clusters.

To understand the program's impact on MAs' workload and job perceptions, we added 2 additional sources of data: a time-motion study of MAs assessing and referring patients, and a qualitative debriefing at the study's conclusion. For the qualitative study, we sampled 2 MAs with high adoption and 2 with low adoption from 5 of the 6 sites. Of 20 planned interviews, 15 were completed. To minimize social desirability bias, MAs were interviewed in private by a research associate who had not been associated with the study. Interviews lasted 40 to 60 minutes. The study team developed a semistructured interview guide focusing on following areas: how MAs perceived their role in the practice, their experience with the referral strategy, including facilitators and barriers; and their experiences, both personal and professional, with health behaviors. Transcripts were read by 3 study team members, who iteratively identified themes and supporting text.

The study was approved by the Institutional Review Board at the University of Texas Health Science Center at San Antonio.

\section{RESULTS}

A total of 864 patients completed the baseline assessment. Figure 2 displays the number of study partici-

\section{Figure 2. Patient flow diagram for randomized trial.}

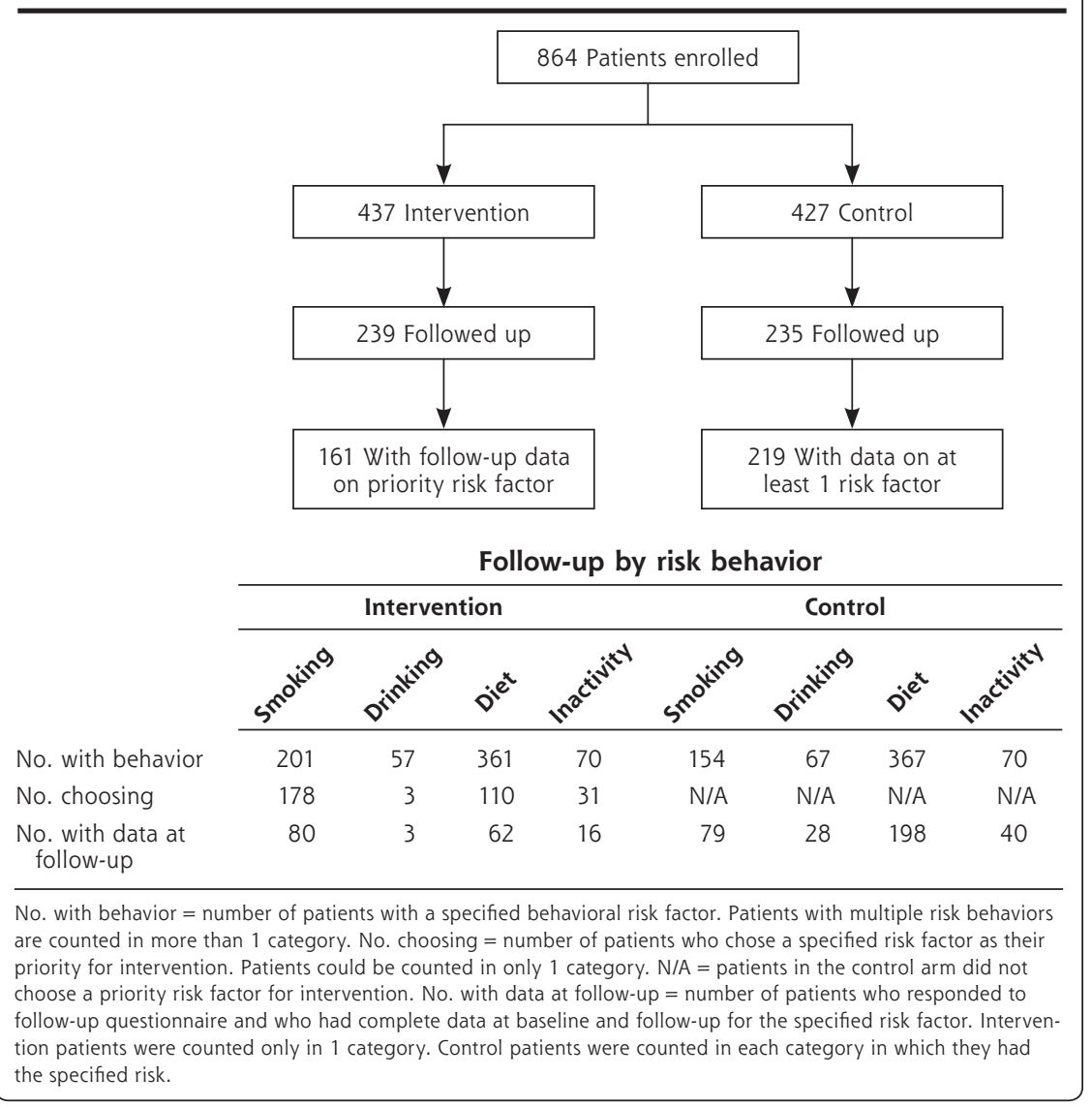


pants in each referral and control group subset: 437 in the MA referral arm and 427 in the control arm. Par- for unhealthy diet, and $75.0 \%$ vs $26.3 \%$ for physical inactivity (all $P<.001$ ). In contrast, no patient attended ticipants' mean age was 46.5 years (range 18 to 75 ). Most were Latino (70.4\%) and economically disadvantaged, with $86 \%$ earning less than $\$ 20,000$ per year. As shown in Table 1, compared with the clinical population from which they were drawn, participants' average age was 5 years younger, and they were more likely to be female and slightly less likely to be Latino. There were no significant differences between MA referral and control groups in age, sex, education, race-ethnicity, or income, though control group participants reported slightly fewer unhealthy days on the CDC Healthy Days measures.

Risk behavior clusters were common, with $62.8 \%$ (95\% confidence interval [CI], 59.6\%-66.1\%) of patients having 2 or more risks (Sup-

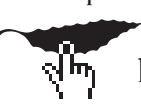
plemental Table 1, available at http://www.annfammed.org/cgi/ content/full/7/6/504/DC1). Smokers with multiple risks commonly chose smoking as their preferred risk behavior for intervention $(80.1 \%$; CI, $72.7 \%-86.3 \%)$. On the other hand, unhealthy diet and physical inactivity were infrequently chosen as priorities unless they occurred in the absence of smoking (Supplemental

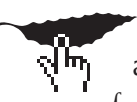

Table 2, available at http://www. annfammed.org/cgi/content/ full/7/6/504/DC1). Risky drinking was rarely sele 25194 cted.

Fifty-five percent of participants completed the follow-up assessment. The median interval was 12 months. Those who completed the assessment were slightly older (47.9 vs 44.9 years; $P<.001)$, more likely to be female ( 77.6 vs $71 \% ; P=.02)$, and less likely to be African American (4.7 vs $10.2 \% ; P=.002$ ).

Patients who were screened and referred by medical assistants for smoking, physical inactivity, or unhealthy diet were much more likely than usual care patients to attend a referral (Table 2): $75.3 \%$ vs $28.6 \%$ for smoking, $60.6 \%$ vs $22.3 \%$

\begin{tabular}{|c|c|c|c|}
\hline Variable & $\begin{array}{l}\text { Referral Program } \\
\qquad N=437\end{array}$ & $\begin{array}{l}\text { Control } \\
N=427\end{array}$ & $\begin{array}{c}\text { Clinical } \\
\text { Population }^{\mathrm{a}}\end{array}$ \\
\hline Age, mean (SD), y & $46.1(11.6)$ & 46.9 (11.9) & 41.0 \\
\hline Female, \% & 73.7 & 75.6 & 57.3 \\
\hline \multicolumn{4}{|l|}{ Race and ethnicity, \% } \\
\hline White, non-Hispanic ${ }^{b}$ & 22.6 & 18.1 & \\
\hline Black, non-Hispanic & 6.8 & 7.7 & 5.2 \\
\hline Hispanic & 68.7 & 71.9 & 76.4 \\
\hline Other or no response $\mathrm{e}^{\mathrm{b}}$ & 1.9 & 2.0 & - \\
\hline Education, \% & & & Not ascertained \\
\hline$<12$ y & 30.5 & 28.0 & - \\
\hline $12 y$ & 35.6 & 39.6 & - \\
\hline$>12$ y & 33.9 & 32.4 & - \\
\hline \multicolumn{4}{|l|}{ Income, \%c } \\
\hline$<\$ 10,000$ & 52.7 & 53.6 & - \\
\hline$\$ 10-19,999$ & 34.1 & 32.5 & - \\
\hline$\$ 20-34,999$ & 10.1 & 11.9 & - \\
\hline$>\$ 35,000$ & 3.1 & 2.1 & - \\
\hline \multicolumn{4}{|l|}{ Risk behaviors, \% } \\
\hline Smoking & 49.5 & 50.5 & - \\
\hline Risky drinking & 18.3 & 18.9 & - \\
\hline Poor diet & 93.8 & 90.0 & - \\
\hline Low physical activity & 28.5 & 25.5 & - \\
\hline Body mass index $\left(\mathrm{kg} / \mathrm{m}^{2}\right)$ & 33.9 & 33.8 & - \\
\hline \multicolumn{4}{|l|}{ Unhealthy days, past $30 \mathrm{~d}$} \\
\hline Physical, mean & 11.7 & 10.1 & - \\
\hline Mental, mean & 12.1 & $9.9^{d}$ & _- \\
\hline
\end{tabular}

Note: Statistical significance evaluated with Fisher's exact test for categorical variables and 2-sample $t$ test for continuous variables. $P>.05$ for all comparisons for referral program vs control group, except for mental unhealthy days.

a Adult enrollees of Bexar County CareLink Program (see Methods for description).

${ }^{\mathrm{b}}$ Target population demographics did not establish these categories separately from Hispanic.

'Most, $99.4 \%$, had income $<200 \%$ of federal poverty limit.

$\mathrm{d} P=.008$

Table 2. Proportion Attending Referral Programs

\begin{tabular}{|c|c|c|c|c|}
\hline Risk Behavior & $\begin{array}{l}\text { Referral } \\
\text { Program } \\
\% \text { (n) }\end{array}$ & $\begin{array}{c}\text { Control } \\
\% \text { (n) }\end{array}$ & $\begin{array}{c}\text { Risk Ratio } \\
(95 \% \mathrm{Cl})\end{array}$ & $\begin{array}{l}\text { Adjusted } \\
\text { Relative Risk } \\
(95 \% \mathrm{Cl})^{a}\end{array}$ \\
\hline Smoking & $75.3(93)$ & $28.6(81)$ & $2.25(1.76-2.62)$ & $2.23(1.52-2.78)$ \\
\hline Alcohol & $0(3)$ & $0(45)$ & - & - \\
\hline Diet & $60.6(66)$ & $22.3(224)$ & $2.30(1.71-2.03)$ & $1.93(1.25-2.60)$ \\
\hline Physical activity & $75.0(16)$ & $26.3(44)$ & $2.72(1.61-3.34)$ & $2.02(0.68-3.17)$ \\
\hline Any risk behavior & $67.4(177)$ & $21.8(394)$ & $<.001$ & - \\
\hline \multicolumn{5}{|c|}{$\begin{array}{l}\text { Note: Percentage of patients with specified risk behavior that attended a referral program and adjusted } \\
\text { relative risks for referral program and control groups. Numbers in parentheses are denominators for com- } \\
\text { parisons (intention to treat). Risk ratios and } 95 \% \text { confidence intervals computed with logistic regression; } \\
\text { odds ratios converted to relative risk ratios by method of Zhang and Yu. } .^{33} \text { Comparison for "any risk behav- } \\
\text { ior" evaluated with } 2 \text {-sample test of proportions. For comparisons of "any risk behavior," control group } \\
\text { patients can contribute denominator data for multiple risk behaviors. }\end{array}$} \\
\hline \multicolumn{5}{|c|}{$\mathrm{Cl}=$ confidence interval. } \\
\hline adjusted for age, se & celetnnicity, & sellne ne & & \\
\hline
\end{tabular}


a referral for risky drinking as a result of the MA protocol. Nevertheless, combining results across the 4 risk behaviors, $67.4 \%$ of patients in the MA referral group vs $21.8 \%$ of patients in the usual care group attended 1 of the risk behavior interventions $(P<.001)$.

No statistically significant differences in behavior change emerged for any of the 4 behaviors (Table 3 ). The few patients in the MA referral arm whose risky drinking patterns improved were referred outside the MA referral protocol. As shown in Tables 2 and 3, adjusting for covariates in multivariable models produced small changes in the risk ratios. Only the risk ratio comparing improvement in physical inactivity crossed a statistical significance threshold as a result of adjustment: the risk ratio was no longer significant.

In the time-motion study, research associates timed 59 MA encounters during the project's late operational phase. MA contact without a study referral averaged 8 minutes (SD, 3.4; mode, 5 minutes). Risk behavior assessment and referral required 5.4 additional minutes (SD, 2.6; mode, 4 minutes). Thus the total time for routine $\mathrm{MA}$ activities plus recruiting, informed consent, and referral averaged 13 minutes.

Overall, the practices' adoption of the MA assessment and referral was poor. We planned for each MA to arrange about 80 referrals, but only 3 of the approximately 100 participating MAs reached that goal.

Supplemental Figure 1, available at http://www.
annfammed.org/cgi/content/full/7/6/504/DC1,

Table 3. Proportion of Patients With Improved Behavioral Outcomes and Adjusted Relative Risks Comparing Referral Program and Control Groups

\begin{tabular}{lcccc}
\hline & $\begin{array}{c}\text { Referral } \\
\text { Program } \\
\%(\mathbf{n})\end{array}$ & $\begin{array}{c}\text { Control } \\
\%(\mathbf{n})\end{array}$ & $\begin{array}{c}\text { Risk Ratio } \\
\mathbf{( 9 5 \% ~ C l )}\end{array}$ & $\begin{array}{c}\text { Adjusted } \\
\text { Relative Risk } \\
(95 \% \mathbf{C l})\end{array}$ \\
\hline Quit smoking & $25.0(80)$ & $21.5(79)$ & $1.16(0.64-1.90)$ & $0.92(0.45-1.64)$ \\
Quit risky drinking & $33.3(3)$ & $14.2(28)$ & - & - \\
$\begin{array}{l}\text { Eating }>5 \text { servings fruit } \\
\text { and vegetables per day }\end{array}$ & $16.1(62)$ & $11.1(198)$ & $1.45(0.71-2.71)$ & $1.91(0.82-3.81)$ \\
Activity >low & $25.0(16)$ & $37.5(40)$ & $0.67(0.22-1.47)$ & $0.63(0.14-1.60)$ \\
Any behavior change & $21.7(161)$ & $16.9(343)^{c}$ & - & - \\
\hline
\end{tabular}

Note: Percentage of patients with specified risk behavior that moved to a lower risk category. Numbers in parentheses are denominators for comparisons (intention to treat). Risk ratios and $95 \% \mathrm{Cl}$ computed with logistic regression; odds ratios converted to relative risk ratios by method of Zhang and Yu. ${ }^{33}$ Comparison for "any risk behavior" evaluated with 2-sample test of proportions. For comparisons of "any risk behavior," control group patients can contribute denominator data for multiple risk behaviors). Definitions of outcomes: quit smoking = current smoker at baseline ( $>100$ cigarettes in lifetime + smoked in past 7 days) but no smoking in past 7 days at follow-up; quit risky drinking = any binge drinking ( 5 or more drinks on 1 occasion) or drinking above threshold ( $>2$ drinks per day for men or $>1$ drink per day for women) in past 30 days at baseline, but neither behavior present in past 30 days at follow-up; eating $>5$ servings fruit or vegetables $=$ consumed $<5$ servings per day of fruits or vegetables in past week at baseline, but exceeded that threshold at follow-up; activity >low = inactive by IPAQ algorithm (using minutes per week of sedentary, low, moderate, and vigorous activity) at baseline, but moderate or high at follow-up.

$\mathrm{Cl}=$ confidence interval; IPAQ = International Physical Activity Questionnaire.

a Adjusted for age, sex, race/ethnicity, and baseline health status.

${ }^{b}$ Risk ratio not computed because no patients selected alcohol referral programs within program.

${ }^{c} P=.19 ; 2$-sample test of proportions. displays the number of referrals arranged by MAs at debriefings at the close of the study, we identified the following major themes: (1) pressure to keep the patient flow moving (from both the MAs themselves and physicians) was a barrier to arranging the refer(2) the extra workload was a challenge ${ }_{i}(3) \mathrm{MA}$ hypocritical delivering health meshindered by their organizations; (5) helping patients change could be very satisfying, perhaps personally more than professionally; and (6) MAs were confident could effectively relate to patients. Illustrative tal Table 3, available at http.//www annfammed. org/cgi/content/full/7/6/504/DC1.

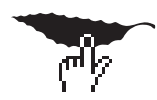

A medical assistant-driven approach to identify 4 major risk behaviors and arrange behavioral interventions was much more effective than usual care at linking patients with interventions, more than doubling the proporwhis finding represents an important extension of the primary care team's ability to intervene for risk behaviors. Despite the process improvement, however, success in changing any of the 4 behaviors did not follow. The dissociation between process and outcomes underscores the difficulty of changing health behaviors. Similar patterns appear in chronic disease management, where behavior change is also critical. ${ }^{35}$ It is also possible that our low- to moderate-intensity interventions failed to motivate lasting change for many patients. ${ }^{36}$

In contrast to the other risk behaviors, risky drinking was almost never selected by patients as their priority, despite its prevalence of $20.4 \%$ in the MA referral group. Beyond patients' reluctance to acknowledge drinking problems, features of the MA strategy may have played a part, as only risky drinking required a 2-step strategy: MAs informed clinicians that a patient chose an 
alcohol treatment, which clinicians were then responsible for negotiating and arranging. Given the good evidence to support brief clinician counseling for problem drinking, ${ }^{37}$ future work should explore what kind of information hand-off from MA to clinician-a written form in this study-can better promote clinicians' effective use of the data.

To differing degrees, practices struggled to adopt the MA referral program. As the debriefing interviews noted, MAs work in series with patient flow through examination rooms, making practices keenly sensitive to potential interruptions. In the research setting, this pressure was intensified by the need for the MAs to recruit patients and obtain their informed consent, tasks that often took longer than the referral program itself. Whether the referral program would have been more fully adopted if it less time had been needed to deliver it remains an open question.

During project startup, we tried to promote program adoption with strategies suggested by practice change models ${ }^{38,39}$ that underscore how critical it is to secure necessary resources and expertise, ${ }^{41}$ cultivate practice champions and leadership, ${ }^{40,42,43}$ and consider stakeholders' perceptions of change in the context of their normative organizational culture. ${ }^{40}$ Two obstacles emerged, however. First, the run-in time and resources were inadequate, constrained in part by the research funding cycle and in part by the limited time practices have available for improvement work. Second, most of the practices lacked the experience and group processes to adapt effectively an innovation to their own circumstances. ${ }^{44}$ In the next iteration, considerably more resources will need to be devoted to MA training, fostering collaboration between MA-physician dyads $^{45}$ to support the referral program, and nurturing a group process for problem solving. ${ }^{46,47}$

$\mathrm{MA}$ roles have been redesigned for many purposes, ${ }^{15,48}$ but smoking cessation has been a frequent focus, most commonly by having MAs record smoking status as a vital sign. ${ }^{49} \mathrm{~A}$ few trials have gone further, with MAs effectively delivering brief counseling messages. ${ }^{17,50}$ To our knowledge, this evaluation is the first of a program in which MAs assess the 4 major behavioral risks, seek agreement, and assist patients with referral programs.

The study has important limitations, many arising from the difficult tradeoffs inherent in a pragmatic, practice-based trial. ${ }^{51}$ Recruitment was subject to 2 biases: MAs could recruit patients at their discretion, effectively enrolling a convenience sample; and MAs were able to anticipate a prospective participant's treatment allocation and thus potentially tamper with randomization. Furthermore, we were unable to track refusal rates and so had to assess participation bias by comparing participants with the clinical population from which they were recruited. The net effect of recruitment and participation bias was that fewer men and slightly fewer Latinos enrolled than expected. Bias in follow-up was also evident, further increasing the proportion of patients who completed the assessment who were women, older patients, and non-Latino whites rather than African Americans. Adjusting for these characteristics, however, did not change the outcomes.

Although the planned sample size allowed for adequate statistical power to assess change for each behavior, the achieved sample size was substantially smaller. As a result, there was diminished power to test small but potentially clinically meaningful changes in health behaviors.

Another important limitation was that behavioral outcomes were self-reported. The relatively high proportion of patients reporting behavior change raises suspicion of social desirability bias, a common concern when measuring health behaviors, though less likely with smoking ${ }^{52}$ than the other behaviors. ${ }^{53,54}$ Although social desirability may have inflated improvement estimates in both study arms, 2 observations suggest that it was not more common in the MA referral arm. First, the effect was not observed for the physical inactivity referrals, in which the control group reported greater improvement. Second, persons who reported improvement for 1 risk behavior were no more likely to report improvement for other behaviors. Lastly, patients may have felt less pressured to report desirable outcomes because follow-up data were collected by research associates rather than the clinical staff that had arranged the referrals.

Missing data and the loss to follow up of $45 \%$ of the sample also create potential biases. As both these problems occurred more commonly among persons with markers of social disadvantage, we likely overestimated the improvement in behavioral outcomes. Taken together, the study's limitations warrant a cautious approach to interpretation; although a randomized trial, it should be viewed as exploratory and suggestive evidence needing confirmation.

Strengths included a novel strategy to broaden team participation in managing health behaviors, standardized behavior change measures, and, perhaps most importantly, using current practice staff rather than adding new personnel. Although the MAs sometimes struggled with the demands of arranging referrals, patients readily accepted the new approach, as shown by their high uptake of referrals. Linking with community resources outside the practices enhanced the practices' capacity to intervene.

Many challenges remain before a strategy such as this can succeed. The interviews with MAs revealed 
the difficulty of redesigning a role that has evolved in a certain direction, ${ }^{55}$ a role occupied by personnel with preexisting expectations of effort reward, who may not have the necessary professional development, ${ }^{56,57}$ and who work in systems that are not enabling them to assume new roles. Human resources, practice design, and organizational change strategies ${ }^{44,58}$ all need to be carefully considered. It is easy to underestimate the time, resources, and staff commitment necessary for sustained practice change. ${ }^{59}$ As the poor effectiveness for behavioral outcomes also showed, further work also needs to focus on developing systems that will support long-term improvements in patients' behaviors. Many of these referral programs will lie outside the practice, but it is essential for them to link tightly to the practice to leverage the relationship that patients have with their primary care team into clinical success.

To read or post commentaries in response to this article, see it online at http://www.annfammed.org/cgi/content/full/7/6/504.

Key words: Practice redesign, behavioral risk factors; medical assistants; primary health care, health promotion; health behavior, allied health occupations

Submitted October 1, 2008; submitted, revised, March 17, 2009; accepted March 24, 2009.

Funding support: The study was funded by the Robert Wood Johnson Foundation's Prescription for Health program, grant No. 053766.

Clinical trials registration No. NCT00273806.

Acknowledgments: Daniel Oyiriaru, MBBS, Lorraine Babcock, RN, and Jenna Becho, BA, provided invaluable assistance with collecting data for this study.

\section{References}

1. McMichael T. Human Frontiers, Environments and Disease: Past Patterns, Uncertain Futures. Cambridge, England: Cambridge University Press; 2001.

2. Mokdad AH, Marks JS, Stroup DF, Gerberding JL. Actual causes of death in the United States, 2000. JAMA. 2004;291(10):1238-1245.

3. Green LA, Fryer GE Jr, Yawn BP, Lanier D, Dovey SM. The ecology of medical care revisited. N Engl J Med. 2001;344(26):2021-2025.

4. Ferrer RL. Pursuing equity: contact with primary care and specialist clinicians by demographics, insurance, and health status. Ann Fam Med. 2007;5(6):492-502.

5. Wagner EH, Austin BT, Von Korff M. Organizing care for patients with chronic illness. Milbank Q. 1996;74(4):511-544.

6. Solberg LI, Kottke TE, Conn SA, Brekke ML, Calomeni CA, Conboy KS. Delivering clinical preventive services is a systems problem. Ann Behav Med. 1997;19(3):271-278.

7. Thorndike AN, Regan S, Rigotti NA. The treatment of smoking by US physicians during ambulatory visits: 1994 2003. Am J Public Health. 2007;97(10):1878-1883.

8. Ferrer RL, Hambidge SJ, Maly RC. The essential role of generalists in health care systems. Ann Intern Med. 2005;142(8):691-699.
9. Stange KC, Woolf SH, Gjeltema K. One minute for prevention: the power of leveraging to fulfill the promise of health behavior counseling. Am J Prev Med. 2002;22(4):320-323.

10. Bar-Yam Y. Multiscale variety in complex systems. Complexity. 2004;9(4):37-45.

11. Bar-Yam Y. Making Things Work: Solving Complex Problems in a Complex World. Brookline, MA: Knowledge Press; 2005.

12. Whitlock EP, Orleans CT, Pender N, Allan J. Evaluating primary care behavioral counseling interventions: an evidence-based approach. Am J Prev Med. 2002;22(4):267-284.

13. Barnes CS, Ziemer DC, Miller CD, et al. Little time for diabetes management in the primary care setting. Diabetes Educ. 2004;30(1): 126-135.

14. Aita V, Dodendorf DM, Lebsack JA, Tallia AF, Crabtree BF. Patient care staffing patterns and roles in community-based family practices. J Fam Pract. 2001;50(10):889.

15. McCarthy BD, Yood MU, Bolton MB, Boohaker EA, MacWilliam $\mathrm{CH}$, Young MJ. Redesigning primary care processes to improve the offering of mammography. J Gen Intern Med. 1997;12(6):357-363.

16. Spencer E, Swanson T, Hueston WJ, Edberg DL. Tools to improve documentation of smoking status. Continuous quality improvement and electronic medical records. Arch Fam Med. 1999;8(1):18-22.

17. Katz DA, Muehlenbruch DR, Brown RL, Fiore MC, Baker TB; AHRQ Smoking Cessation Guideline Study Group. Effectiveness of implementing the agency for healthcare research and quality smoking cessation clinical practice guideline: a randomized, controlled trial. J Natl Cancer Inst. 2004;96(8):594-603.

18. Thompson RS, Meyer BA, Smith-DiJulio K, et al. A training program to improve domestic violence identification and management in primary care: preliminary results. Violence Vict. 1998;13(4):395-410.

19. Grumbach K, Bodenheimer T. Can health care teams improve primary care practice? JAMA. 2004;291(10):1246-1251.

20. Bodenheimer T, Laing BY. The teamlet model of primary care. Ann Fam Med. 2007;5(5):457-461.

21. Cifuentes M, Fernald DH, Green LA, et al. Prescription for health: changing primary care practice to foster healthy behaviors. Ann Fam Med. 2005;3(Suppl 2):S4-S11.

22. Glasgow RE, Ory MG, Klesges LM, Cifuentes M, Fernald DH, Green LA. Practical and relevant self-report measures of patient health behaviors for primary care research. Ann Fam Med. 2005;3(1):73-81.

23. US Department of Health and Human Services. 2004. Behavioral Risk Factor Surveillance System. http://www.cdc.gov/brfss/questionnaires/pdf-ques/2004brfss.pdf.

24. Craig CL, Marshall AL, Sjöström M, et al. International physical activity questionnaire: 12-country reliability and validity. Med Sci Sports Exerc. 2003;35(8):1381-1395.

25. Ammerman AS, Haines PS, DeVellis RF, et al. A brief dietary assessment to guide cholesterol reduction in low-income individuals: design and validation. J Am Diet Assoc. 1991;91(11):1385-1390.

26. US Department of Health and Human Services, Centers for Disease Control and Prevention, National Center for Chronic Disease Prevention, Division of Adult and Community Health. Measuring Healthy Days: Population Assessment of Health-Related Quality of Life. Atlanta, GA: US Department of Health and Human Services; 2000.

27. Bandura A. Human agency in social cognitive theory. Am Psychol. 1989;44(9):1175-1184.

28. Ornstein SM, Musham C, Reid A, Jenkins RG, Zemp LD, Garr DR. Barriers to adherence to preventive services reminder letters: the patient's perspective. J Fam Pract. 1993;36(2):195-200.

29. Zhu SH, Anderson CM, Tedeschi GJ, et al. Evidence of real-world effectiveness of a telephone quitline for smokers. $N$ Engl J Med. 2002;347(14):1087-1093. 
30. Brownson RC, Smith CA, Pratt M, et al. Preventing cardiovascular disease through community-based risk reduction: the Bootheel Heart Health Project. Am J Public Health. 1996;86(2)206-213.

31. Thomson CA, Giuliano A, Rock CL, et al. Measuring dietary change in a diet intervention trial: comparing food frequency questionnaire and dietary recalls. Am J Epidemiol. 2003;157(8):754-762.

32. Kaner EF, Beyer F, Dickinson HO, et al. Effectiveness of brief alcohol interventions in primary care populations. Cochrane Database Syst Rev. 2007;Apr 18(2): CD004148.

33. Zhang J, Yu KF. What's the relative risk? A method of correcting the odds ratio in cohort studies of common outcomes. JAMA. 1998;280(19):1690-1691.

34. Schafer JL. Multiple imputation: a primer. Stat Methods Med Res. 1999;8(1):3-15.

35. Landon BE, Hicks LS, O'Malley AJ, et al. Improving the management of chronic disease at community health centers. $N$ Engl J Med. 2007;356(9):921-934.

36. Marcus BH, Dubbert PM, Forsyth LH, et al. Physical activity behavior change: issues in adoption and maintenance. Health Psychol. 2000;19(1)(Suppl):32-41.

37. Moyer A, Finney JW, Swearingen CE, Vergun P. Brief interventions for alcohol problems: a meta-analytic review of controlled investigations in treatment-seeking and non-treatment-seeking populations. Addiction. 2002;97(3):279-292.

38. Cohen D, McDaniel RR, Crabtree BF, Ruhe MC, Weyer SM. al E. A practice change model for quality improvement in primary care practice. J Healthc Manag. 2004;49(3):155-168.

39. Cohen DJ, Tallia AF, Crabtree BF, Young DM. Implementing health behavior change in primary care: lessons from prescription for health. Ann Fam Med. 2005;3(Suppl 2):S12-S19.

40. Berwick DM. Disseminating innovations in health care. JAMA. 2003;289(15):1969-1975

41. Bradley EH, Webster TR, Baker D, et al. Translating Research Into Practice: Speeding the Adoption of Innovative Health Care Programs. New York, NY: The Commonwealth Fund. July, 2004

42. Kottke TE, Solberg LI. STEP(ing)-UP to deliver clinical preventive services. Am J Prev Med. 2001;21(1):68-69.

43. Ozer EM, Adams SH, Lustig JL, et al. Increasing the screening and counseling of adolescents for risky health behaviors: a primary care intervention. Pediatrics. 2005;115(4):960-968.

44. Yano EM, Rubenstein LV, Farmer MM, et al. Targeting primary care referrals to smoking cessation clinics does not improve quit rates: implementing evidence-based interventions into practice. Health Serv Res. 2008 Jun 3. [Epub ahead of print].
45. Crabtree BF, McDaniel RR, Nutting PA, Lanham HJ, Looney AJ, Miller WL. Closing the physician-staff divide: a step toward creating the medical home. Fam Pract Manag. 2008;15(4):20-24.

46. Solberg LI, Brekke ML, Fazio CJ, et al. Lessons from experienced guideline implementers: attend to many factors and use multiple strategies. Jt Comm J Qual Improv. 2000;26(4):171-188.

47. Solberg LI, Hroscikoski MC, Sperl-Hillen JM, Harper PG, Crabtree BF. Transforming medical care: case study of an exemplary, small medical group. Ann Fam Med. 2006;4(2):109-116.

48. Bass MJ, McWhinney IR, Donner A. Do family physicians need medical assistants to detect and manage hypertension? Can Med Assoc J. 1986;134(11):1247-1255.

49. Solberg LI. Incentivising, facilitating, and implementing an office tobacco cessation system. Tob Control. 2000;9(Suppl 1):137-41.

50. Katz DA, Muehlenbruch DR, Brown RB, Fiore MC, Baker TB; AHRQ Smoking Cessation Guideline Study Group. Effectiveness of a clinicbased strategy for implementing the AHRQ Smoking Cessation Guideline in primary care. Prev Med. 2002;35(3):293-301.

51. Cohen DJ, Crabtree BF, Etz RS, et al. Fidelity versus flexibility: translating evidence-based research into practice. Am J Prev Med. 2008;35(5)(Suppl):S381-S389.

52. Patrick DL, Cheadle A, Thompson DC, Diehr P, Koepsell T, Kinne S. The validity of self-reported smoking: a review and meta-analysis. Am J Public Health. 1994;84(7):1086-1093.

53. Hebert JR, Clemow L, Pbert L, Ockene IS, Ockene JK. Social desirability bias in dietary self-report may compromise the validity of dietary intake measures. Int J Epidemiol. 1995;24(2):389-398.

54. Midanik LT. Validity of self-reported alcohol use: a literature review and assessment. Br J Addict. 1988;83(9):1019-1030

55. Tache S, Chapman S. The expanding roles and occupational characteristics of medical assistants: overview of an emerging field in allied health.. J Allied Health. 2006;35(4):233-237.

56. Katz DA, Brown RB, Muehlenbruch DR, Fiore MC, Baker TB; AHRQ Smoking Cessation Guideline Study Group. Implementing guidelines for smoking cessation: comparing the efforts of nurses and medical assistants. Am J Prev Med. 2004;27(5):411-416.

57. Braun BL, Fowles JB, Solberg LI, Kind EA, Lando H, Pine D. Smoking-related attitudes and clinical practices of medical personnel in Minnesota. Am J Prev Med. 2004;27(4):316-322.

58. Stone EG, Morton SC, Hulscher ME, et al. Interventions that increase use of adult immunization and cancer screening services: a meta-analysis. Ann Intern Med. 2002;136(9):641-651.

59. Grol R. Personal paper. Beliefs and evidence in changing clinical practice. BMJ. 1997;315(7105):418-421. 